\title{
El republicanismo gallego en Chile: las Crónicas de Suárez Picallo
}

\author{
The Galician republicanism: The Suárez Picallo’s Chronicles
}

\author{
Constanza MANRíQuez Bergenfreid \\ Andrés SuÁrez GonZález \\ Universidad Santiago de Chile \\ Programa de Estudios Gallegos. Instituto de Estudios Avanzados \\ conti.manriquezb@gmail.com \\ andres.suarez@usach.cl
}

[recibido 28/11/2011, aceptado 07/03/2012]

\section{RESUMEN}

El artículo analiza la figura del intelectual de origen gallego Ramón Suárez Picallo (1894-1964) y algunas de sus crónicas escritas en Chile entre 1942 y 1956 y recopiladas en el libro La feria del mundo (2008). Una de las motivaciones de sus crónicas sería el exilio republicano en América, especialmente el gallego. Suárez Picallo señala a Chile y a otros países de América Latina como un ejemplo político para el republicanismo español.

PALABRAS ClaVE: La feria del mundo, Ramón Suárez Picallo, exilio gallego, republicanismo español.

Manríquez Bergenfreid, C. y SuÁrez GonzÁLez, A. (2012): "El republicanismos gallego en Chile: las Crónicas de Suárez Picallo", Madrygal (Madr.), 15: 87-95.

\section{RESUMO}

O artigo fai unha análise do intelectual de orixe galega Ramón Suárez Picallo (1894-1964) e algunhas das súas crónicas escritas en Chile entre 1942 e 1956 e recompiladas no libro La feria del mundo (2008). Unha das motivacións das súas crónicas sería o exilio republicano en América, especialmente o galego. Suárez Picallo sinala Chile e outros países de América Latina como un exemplo político para o republicanismo español.

PALABRAS ChaVE: La feria del mundo, Ramón Suárez Picallo, exilio galego, republicanismo español.

Manríquez Bergenfreid, C. e SuÁrez GonzÁlez, A. (2012): “O republicanismo galego en Chile: as Crónicas de Suárez Picallo", Madrygal (Madr.), 15: 87-95.

\begin{abstract}
This article examines the figure of the galician intelectual Ramón Suárez Picallo (1894-1964) and some of the chronicles that he wrote in Chile between 1942 and 1956 and that were compiled in the book La feria del mundo (2008). One of his motivations for having written the chronicles may have been his republican exile in America, especially his galician exile. Suárez Picallo points to Chile and to other Latin American countries as a political example for Spanish republicanism.
\end{abstract}

KEY WORDS: La feria del mundo, Ramón Suárez Picallo, Galician exile, Spanish republicanism.

Manríquez Bergenfreid, C. and SuÁrez GonzÁlez, A. (2012): "The Galician republicanism: The Suarez Picallo's Chronicles", Madrygal (Madr.), 15: 87-95. 
SUMARIO: 1. Introducción. 2. El autor. 3. Las crónicas de Suárez Picallo y el republicanismo alén do mar. 4. A modo de conclusión. 5. Referencias bibliográficas.

\section{INTRODUCCIÓN}

Existen dos intelectuales de origen gallego con fuerte presencia en el mundo cultural de la primera mitad de siglo XX en Chile: Eduardo Blanco Amor y Ramón Suárez Picallo. Su arribo a Chile se produce dentro del contexto de ola migratoria española en el país durante el siglo XX que, alrededor de 1939, según palabras de Estrada Turra (2009) puso en dos bandas a los españoles residentes con la llegada de exiliados y, "la diferencia de otros lugares, como México y Argentina, en donde también arribaron exiliados y se produjeron conflictos, la confrontación entre ambos bandos, en Chile, comenzó desde antes de la llegada de los exiliados" (Estrada Turra 2009: 96). El punto de inflexión de este proceso fue la llegada del barco Winnipeg a Chile. Sobre la cantidad de exiliados republicanos, Estrada Turra señala "que llegaron a Chile en un número cercano a los 3500 , de los cuales unos 700 se establecieron en Valparaíso" (Estrada Turra 2009: 96).

En lo que se refiere a los emigrantes de origen gallego a lo largo de la historia de Chile, esta se encuentra aún poco documentada y caracteriza$\mathrm{da}^{1}$, como sí ocurre respecto de otros países de América Latina como Argentina, Brasil, Cuba, Uruguay o Venezuela ${ }^{2}$. Según señala Villares "Chile no estuvo jamás en el centro de un proceso de inmigración masiva" (1996: 135). Los flujos migratorios, en el caso de este país, hasta la segunda mitad del siglo XIX estuvieron concentrados en población de origen alemán, inglés y francés, con un carácter agrícola (Sánchez Espinoza 1995). Hacia 1880 se trató de diversificar esta emigración. Y en 1885 , como señala Villares, citando a Norambuena, "la fuerte emigración española hizo que esta colectividad ocu- para el primera lugar" (Villares 1996: 136). Hacia 1930 el flujo migratorio hacia Chile tenía un origen principalmente español, italiano y alemán (Suárez González 2011).

Como se mencionaba al inicio, un punto simbólico de la emigración española a Chile en el siglo XX fue marcado por el arribo del barco Winnipeg, en septiembre de 1939, cargado de refugiados republicanos de la Guerra Civil, que incluía a hombres, mujeres y niños. Según documenta un texto editado por la Fundación Winnipeg en Chile, la gestión del barco ocurrió de la siguiente manera:

A instancias de [Pablo] Neruda, el gobierno Republicano en el exilio, a través del Servicio de Evacuación de Refugiados Españoles (S.E.R.E.), había contratado el vapor Winnipeg a la Compagnie France-Navigation para el traslado de unos 2.000 refugiados a Chile [...] El tema dividió ideológicamente a la población. [...] las disputas hicieron tambalear al gabinete de Pedro Aguirre Cerda y alcanzaron a la misma Cámara de Diputados poco antes del zarpe del Winnipeg desde el puerto fluvial de Pauillac. (Ferrer Mir 1999: 12)

El tres de septiembre de 1939 arriban al puerto de Valparaíso 2365 personas (Ferrer Mir 2004), entre ellos, notables intelectuales y artistas que contribuirían al país sudamericano. Como bien señala Norambuena "[siendo] importante en lo intelectual, no alcanzaba los guarismos de la emigración alemana, pero que sin embargo representaba un esfuerzo significativo en el Chile de los 50 en adelante" (1999: 21).

Como en el resto de los países latinoamericanos, ya hacia los años cincuenta se estanca el proceso migratorio hispano hacia $\mathrm{Chile}^{3}$, con importantes aportes de figuras que adherían al republicanismo; una de ellas fue la del gallego Ramón Suárez Picallo. Su visión sobre Chile, España y las ideas políticas de los españoles en América Latina podemos conocerla por la edición

\footnotetext{
${ }^{1}$ Al respecto, puede verificarse la presencia gallega en algunas familias fundacionales de Chile. Véase Retamal, Atria y Muñoz Correa (1992) y Thayer Ojeda (1989).

${ }^{2}$ Al respecto, cabe señalar que en el "Primeiro Congreso da Emigración Galega" de 1956, tres entidades gallegas de Chile de las ciudades de Santiago, Valparaíso y Punta Arenas se hicieron presente en el encuentro celebrado en Buenos Aires (Congreso da Emigración Galega, 1956)

${ }^{3}$ Haciendo un breve paréntesis, la presencia actual de ciudadanos españoles se puede observar a partir del número de votantes inscritos en el Censo Electoral de Residentes en el Extranjero (CERA), que en noviembre de 2011 informa de que en Chile están inscritos 35774 electores (Boletín Oficial del Estado n 286). Por otro lado, de acuerdo al Censo Electoral de Gallegos en el exterior de 2010 , los países de la región con mayores votantes son Argentina con 125 697, Venezuela con 35 086, Brasil con 31490 y Uruguay con 31 059. Otros países de menor representatividad son Cuba con 15 311, México con 8341, Panamá con 1995 o Chile, en donde hay 1894 de origen gallego. De acuerdo con el Instituto Galego de Estadística, en 2011 se contaba con 2725 personas de nacionalidad española de origen gallego en Chile. Sin embargo, y este es un dato a trabajar en el futuro, hay familias de origen gallego que al emigrar a Chile
} 
de La feria del mundo (2008), que relata sus crónicas periodísticas entre 1942 y 1956 en este país. En este mismo año, durante el "Primeiro Congreso da Emigración Galega", el autor, junto a Marcial Fernández, presentó una declaración política en Buenos Aires en la que hizo un llamado a los emigrados a "luchar por la libertad de los gallegos" (Congreso da Emigración Galega 1956: 195). En este contexto, Chile y otros países de la región latinoamericana serán un ejemplo de liberación y de esperanza ideológica.

\section{EL AUTOR}

Ramón Suárez Picallo (Sada, 1894-Buenos Aires, 1964) fue un intelectual comprometido con la reivindicación de la España republicana y la imagen de Galicia como ideario político y social. Fuera de su país natal desde muy joven, se mantuvo fuertemente vinculado con sus raíces gallegas mediante la participación en distintas instituciones y medios escritos de España y América. Emigró a Buenos Aires en 1912 con el propósito de crear fortuna dentro de un masivo proceso migratorio que tuvo lugar entre fines del siglo XIX y principios del XX. Radicado en Argentina, se desenvolvió en labores afines a la pescadería, concentrándose luego en funciones sindicales y sociales que definieron su pensamiento y acción política a partir del contacto que estableció en esos años con el mundo obrero 4 . De ahí que no sorprenda su militancia en el Partido Socialista Argentino y su intervención en ciertos espacios de discusión relativos a los derechos de los trabajadores. Seguida y paralelamente, Suárez Picallo se desarrolló como periodista en la prensa gallega de España y Argentina, aportando artículos de corte político orientados a la difusión de la ideología republicana y su conjunción con la autonomía de Galicia. Retornó a su tierra natal durante la Segunda República, volviéndose representante del Partido Galleguista y también estudiante de derecho en la Universidad de Santiago de Compostela. Tal estadía se vio frustrada por la irrupción de la Guerra Civil Española cuando se encontraba en Madrid y su posterior marcha a
Francia, desde donde viajó a América del Norte, morando en Estados Unidos y en la zona central del continente hasta instalarse de forma definitiva en Chile a principio de los años $40^{5}$. Una vez situado en la capital del país de acogida, destacó por su columna "La feria del mundo", publicada principalmente en el periódico La Hora, y por su contribución a organizaciones republicanas y gallegas constituidas desde el exilio.

\section{LAS CRÓNICAS DE SUÁREZ PICALLO Y EL REPUBLICANISMO ALÉN DO MAR}

Dentro de la labor periodística de Ramón Suárez Picallo, resalta La feria del mundo. Crónicas desde Chile (1942-1956) (2008), compendio de sus crónicas escritas en la prensa chilena, editado por Carmen Norambuena y Edmundo Moure y objeto de la presente investigación. Se trata, como bien señalan los compiladores, de un conjunto de textos que abordan una infinidad de temas y motivos, entre ellos, la crítica a la España Franquista y a la Europa Nazi, la división ideológica enardecida por la Segunda Guerra Mundial, la mitificación de Galicia y sus raíces celtas, la configuración del republicanismo en el exilio, los elogios a América por sus instituciones democráticas, la reseña de eventos artísticos de diversas disciplinas en espacios chilenos, la preparación de productos marinos, el metaperiodismo, etc. ${ }^{6}$. Es, sin duda, una obra que aúna distintas materias, lo que se explica por el carácter periódico de la publicación y la propensión de este tipo de escritura hacia el impresionismo sobre la cotidianeidad social en la que se inserta. Ello no quiere decir que no puedan encontrarse líneas transversales de lectura sobre este corpus textual, pues, pese a su variedad, puede afirmarse que existe una redundancia de reflexiones que lo integran y definen, tocantes a la situación del destierro y las implicancias que esta manifiesta en la integración a una nueva sociedad. De aquí que se ensaye una lectura relacionada con el conflicto que significa para Suárez Picallo el concebirse como un exiliado y cierta tendencia a identificar la Segunda República con la democracia de

fueron empadronadas en España en ciudades distintas a las de origen, por lo que la cantidad de españoles de origen gallego sería mayor. La línea más investigada sobre emigración gallega a Chile es la del pueblo de Chaguazoso, cadena migratoria documentada en Sánchez Espinosa (1995).

4 Aspectos biográficos de Ramón Suárez Picallo en Cornejo Serrano (2006).

5 Sobre el exilio republicano en Chile, véase Norambuena y Garay (2002).

6 En Suárez Picallo (2008: 27-37). 
América en una suerte de oposición a los gobiernos opresores creados y repetidos a lo largo de la historia por determinados sectores de Europa. En otras palabras, se atiende a la tensión, recurrente en las crónicas, entre la añoranza por una España autónoma, democrática y republicana y su proyección en la tierra de acogida como un ideal factible capaz de alzarse a modo de ejemplo para recuperar el orden perdido en la Península Ibérica. Así, observa en La feria del mundo. Crónicas desde Chile (1942-1956), la configuración de un sujeto fronterizo que se enfrenta a la condición de exiliado mediante la búsqueda de una identidad colectiva ${ }^{7}$ que, al tiempo de homologar la opresión de los totalitarismos en España y América para la creación de un imaginario común, se constituye como la reedificación de un pensamiento ideológico y una esperanza política frente al desolador panorama europeo de aquellos años.

Xosé Manoel Núñez Seixas, estableciendo las particularidades del exilio gallego dentro del exilio republicano y antifranquista, ubica a Suárez Picallo en la categoría "o exiliado por azar xeográfico", referente a los sujetos esparcidos por España (intelectuales, sindicalistas, trabajadores en cuerpos de administración y organizaciones españolas, estudiantes, etc.) a los que la Guerra Civil encontró en distintas zonas del frente lejanas a Galicia y que tuvieron que sumarse a grupos de combate para sobrevivir, la mayoría emigrantes intrapeninsulares, estacionales o permanentes. También entran aquí aquellos que viviendo no solo fuera de Galicia sino también de España, al estallar la Guerra Civil, su posicionamiento antifascista no les habría permitido regresar una vez instalado Franco en el poder (Núñez Seixas 2006: 25-29). Si bien el exilio de Suárez Picallo en Chile no es inmediato a su salida de Francia, es posible considerarlo parte del grupo que llega a este país del sur de América motivado por el gobierno de Pedro Aguirre Cerda y la instancia del Frente Popular, coalición afín a la República española. Dicho grupo fue el más amplio de la inmigración española en tierras chilenas ${ }^{8}$, pues, además de los refugiados del Winnipeg, muchos otros republicanos arribaron mediante transportes alternativos y en fechas sucesivas al fin de la
Guerra Civil. Este colectivo se regeneró en Chile a través de eventos y reuniones sociales y, en ello, Suárez Picallo fue fundamental ya que desde la prensa se preocupó de enaltecer la imagen de la República asemejándola a un ideal democrático. Es más, Chile se construye en el discurso del autor como un lugar que, junto a gran parte de América, representa una unidad tanto en lo político como en lo geográfico y lo espiritual. Son reiteradas las alabanzas al país y al continente por su moralidad y el respeto a la legitimidad de los poderes y las instituciones, como muestran las crónicas "Entendimiento cordial" y "Universidad y democracia". A ello se suman los elogios a lo autóctono y a las raíces indígenas, como ocurre en "América", "Araucanía" y "El mundo es ancho y ajeno". De esta manera, se dibuja una semejanza entre la República y el continente americano que le permite al sujeto trazar una esperanza sobre su exilio y el devenir del mundo político como enmienda a la pérdida general dejada por la Guerra Civil.

Una de las crónicas que tratan el agradecimiento al país de acogida y la percepción de Suárez Picallo sobre sí mismo como sujeto exiliado, es "Homenaje a la prensa chilena". En este reconocido texto, el autor celebra la existencia de La Aurora de Chile y de fray Camilo Henríquez, su fundador y director, subrayando el periodo en que se luchaba por la libertad e independencia de América y en que la prensa se utilizaba como vehículo de las ideas. Cabe señalar a este respecto, como plantean Carmen Norambuena y Cristián Garay, la importancia de la prensa como actividad laboral común en los exiliados profesionales: "El empleo de actividades conexas o propias del periodismo es una de las áreas importantes de la emigración intelectual republicana" (2002: 222). Suárez Picallo asegura ejercer con gusto el periodismo en este país junto a los continuadores de La Aurora de Chile, el primer periódico chileno, por ser la chilena una prensa progresista y expresión de la universalidad de su pueblo, además del retrato de una cultura y espiritualidad que la convierten en el alma de una democracia integral. Sin embargo, pese a la imagen positiva que domina su pensamiento acerca de Chile, su integración se ve frenada por el fan-

\footnotetext{
${ }^{7}$ Al hablar de identidad colectiva o nación, se acoge la visión presente en Anderson (1993).

8 Para mayor información de antecedentes históricos al exilio español en Chile, véase Almonacid Zapata (2004).
} 
tasma del exilio, como se deja ver en una de sus más emblemáticas citas:

Para quien ama, como a la propia luz de sus ojos, a la tierra en que nació y sobre la cual hizo su espíritu el primer aprendizaje de belleza y de amor, hay una tremenda y dramática palabra que resume todas las desventuras. «Desterrado», es la expresión de desconsuelo, de angustia y de amargura, que gravita hoy sobre miles de almas, alejadas de su medio propio. No hay para el dolor que las agobia posibles paliativos. Ni la hospitalidad, ni el afecto, ni la estimación, ni el pan, ni el vino ofrecidos sobre la mesa extendida, tienen fuerza bastante para hacerles olvidar su triste condición de desterrados; es decir, de apartados de su propia tierra, de su hogar y del camposanto donde reposan sus muertos queridos. (Suárez Picallo 2008: 48-49)

El texto da cuenta de cómo el destierro no permite al sujeto integrarse del todo en la sociedad de acogida aún cuando la recepción es amable; es decir, la condición de ser un exiliado se inscribe en Suárez Picallo de tal forma que debe vivir con ello como si se tratase de un estigma. Esta idea es aclarecida por Alicia Alted Vigil, quien, en alusión a la emigración forzosa republicana, afirma:

El exilio implica un desplazamiento existencial, espiritual y geográfico. Desde el momento en que el exiliado abandona forzadamente su país, el entorno vital en el que se construyó su identidad de origen, se ve abocado a convivir con los sentimientos del desarraigo y la nostalgia. (2006: 70)

Es esa autodefinición, o bien la imposibilidad de arraigarse a una tierra distinta a la que se pertenece por origen, la que lo lleva a buscar una identidad nueva que dé estructura a aquello que ya no lo tiene. Es, quizás, para curar el dolor de sentirse un expulsado que el autor se aferra a la imagen de un pueblo oprimido que pugna por su libertad, evocando un tránsito de diferente suerte al sufrido por la Segunda República. De cierta forma, el articularse como exiliado lo conduce a asimilar el sufrimiento de su grupo con el de los americanos frente a un enemigo común que representa la represión y el despotismo. Se trata de reunir dos marginalidades, la República, por un lado, y el mundo latinoamericano, por el otro, entre las que el autor se sitúa, para crear un modelo político e identitario a partir del cual pueda conformarse, generar sentido y, al mismo tiempo, resistir la desalentadora expansión del fascismo en la Europa de entonces. No sorprende, por lo tanto, que en esta misma crónica aluda a la dificultad de ejercer su oficio teniendo en cuenta lo que ocurre en el exterior, vale decir, el problema de tener que abordar acontecimientos históricos regidos por el absurdo y las contradicciones.

El punto de partida de la búsqueda de esa nueva identidad se halla en "Galicia", texto que rememora la decapitación en la plaza pública de Mondoñedo del Mariscal Pedro Pardo de Cela, señor del Valle del Oro, en 1483. Aquí se cuenta la historia de este gallego condenado a muerte por el juez Chinchilla a nombre de la Reina Isabel la Católica por comandar la resistencia de su pueblo al imperio. Para Suárez Picallo este hecho es fundamental, ya que es esa muerte la que hace a Galicia perder su "personalidad nacional, mantenida en tiempos de los celtas, los romanos, los suevos y las monarquías cristianas galaico-asturleonesas" (2008: 113). La crónica aborda la figura de Pardo de Cela, quien renuncia a su grado de Mariscal de Campo del rey don Juan II en un acto de solidaridad con los procuradores de las villas gallegas libres que protestaban ante el monarca, debido a que los nobles feudales cometían desafueros en su tierra contra expresos documentos reales. Dicha protesta no es admitida por el Rey, lo que lleva al Mariscal a retirarse a su castillo de A Frouseira en el Valle de Oro (hoy Santa Cilla de Valadouro). Suárez Picallo cuenta que luego de la muerte de Enrique IV hubo conflictos con la sucesión de la corona de Castilla, a causa de ciertos rumores que aseguraban que el Rey era impotente y que su hija, doña Juana, no era su heredera legítima. Ello condujo a que su hermana Isabel la Católica, disputara la corona mediante una guerra civil de la que salió airosa, mientras Juana, de quien eran partidarios los gallegos, incluido Pardo de Cela, aceptó su derrota recluyéndose en un monasterio. Sin embargo, en Galicia la resistencia siguió férrea durante 14 años, hasta la decapitación del Mariscal. Suárez Picallo explica esta lucha apuntando:

Terminada la guerra en todo el resto de España, coronada Isabel y recluida doña Juana, Galicia, con Pedro Pardo a la cabeza, siguió la resistencia por espacio de 14 años. Porque, para Galicia, la lucha tenía más largos alcances que la defensa de una Princesa que le era amada. Se jugaba, en el pleito, el mantenimiento de su vieja personalidad histórica, como país con lengua, leyes, usos y costumbres; y su unión con Portugal, tierra 
hermana, racial, geográfica, lingüística y espiritualmente. (Suárez Picallo 2008: 114)

Para Galicia, la derrota de Juana significó una serie de castigos que cambiaron para siempre su identidad como pueblo, pues, además de privarla de votos y generar emigración forzosa, se prohibió su lengua y se la desvinculó de sus raíces. El trágico final de Pardo de Cela, quien es decapitado junto a su hijo pese a ser previamente indultados por la Reina, funciona simbólicamente como la pérdida de una conciencia grupal y de la pluralidad como elemento constitutivo de la nación. Sobre el nacionalismo de Suárez Picallo, Cornejo Serrano, además de subrayar la mitificación de la presencia celta y romana en Galicia que hace el autor de las crónicas, hace especial mención al uso de la historia en la narración de lo nacional: "en su discurso sobre Galicia como nación, al igual que los principales teóricos galleguistas, recurre a la historia para explicar el ser diferencial de su tierra con respecto a los demás pueblos ibéricos y del Estado Español" (2006: 383). El sometimiento de Galicia por la fuerza en pos de la unificación española que defendían los monarcas, puede interpretarse como el origen de un retraso cultural y de una difícil economía de los que no alcanzó a recuperarse cuando Franco, en clara afinidad con los Reyes Católicos, volvió a anular su diferencia.

En este sentido, cobra interés en Suárez Picallo la idea del enemigo común sobre gallegosrepublicanos y americanos, ya que fueron los mismos Reyes los que oprimieron a ambos pueblos y condicionaron irremediablemente su futuro. La aparición de los fascismos del siglo XX, parece repetir una vieja historia de la que, para Suárez Picallo, no se aprendió nada. De ahí su preocupación constante por América como ejemplo de Repúblicas democráticas que supieron revelarse contra la tiranía europea, imagen que lleva a la situación gallega en la búsqueda de identidad y esperanza. La tensión entre el deseo de revivir una Galicia Republicana y su constante proyección en el mundo americano y chileno como modelo plausible de gobierno para España, más que resolverse, se articula como una suerte de imaginario de pueblo sometido y/o marginal en oposición a los totalitarismos hegemónicos de la historia de Europa. Son muchas las crónicas en las que se bosqueja y caricaturiza al enemigo fascista, ya sea desde el humor o la rabia, como se aprecia en "El judío fugitivo", "Fue para llegar a eso",
"Inhabilitados los monárquicos" o "La Falange española y el fascismo italiano". Al autor no deja de sorprenderle ni dolerle lo que ocurre en su tierra natal pese a sentirse cómodo en el país de acogida, y ello lo fragmenta en esas dos realidades, pues, como exiliado continúa con su compromiso republicano a la espera de que su gobierno se reinstale en España y, al mismo tiempo, se inserta en una sociedad que le otorga las cualidades democráticas a las que aspira pero que no termina de bastarle por no tratarse de su país de origen. El sujeto se halla entonces frustrado en cuanto a su sentido de pertenencia, y es ese desarraigo el que determina la necesidad de crear una noción de unidad grupal que, recogiendo las diferencias, se trace en función de un ideal compartido capaz de reordenar la esperanza de un mundo justo e igualitario.

Estas ideas se esclarecen en la crónica " 14 de abril, la República española y el día de las Américas", texto en el que Suárez Picallo pretende relacionar la conmemoración del día de las Américas con la proclamación de la Segunda República. El autor define la lucha por la independencia americana como una guerra civil entre liberales, republicanos y monárquicos absolutistas, y ello se vincula al caso español en cuanto la República, como una de las naciones colonizadoras, intenta plegarse al modelo político libertario adoptado por las que fueron sus colonias. Para el autor, lo que sostiene la personalidad de los pueblos americanos independientes es la elección de la democracia en contraposición a las metrópolis absolutistas, al igual que la República en tanto sistema alzado en contra de las tradiciones españolas y europeas que han contribuido a la creación de miseria, desigualdad y barbarie en distintas épocas y continentes. El llamado de Suárez Picallo se dirige hacia una noción de aprendizaje que, ya ensayada por el sistema de izquierda, incita a España a acoger los propósitos de América, como muestra la siguiente reflexión:

España debería tener una posición política y diplomática, concordante con las naciones de su estirpe. No tiene esa posición, sino la contraria. La España actual, es todo un problema en relación con la posición, la conveniencia y la lucha democrática de las naciones de América en el actual conflicto bélico. Una España republicana, reverso de la medalla, sería en cambio, para América, en la guerra, en las post-guerra y en la paz, un centro de amistad y un sólido punto de apoyo. Por algo, fue la República Española, por lo 
que era ella y por lo que significaba en la geografía y en la Historia del mundo, el primer país atacado antes de iniciar el nazifascismo, su carrera vertiginosa de invasiones, de arrasamientos y sometimientos de naciones y pueblos cultos y soberanos. (Suárez Picallo 2008: 226)

La crítica al papel de España en la Segunda Guerra Mundial, enfatiza la diferencia ideológica que divide el mundo de entonces para sugerir que la consolidación de los fascismos europeos cimentó sus raíces en la Península Ibérica. En este punto, conviene mencionar los postulados de Rosario Alonso García, quien afirma que la República no se reinstauró en España por el panorama ideológico de la posguerra, ya que, el lugar geográfico y políticamente estratégico que ocupaba el país en Europa y el momento histórico que se vivía, motivaron a Gran Bretaña y Estados Unidos a una política de "no intervención" para no dar cabida a un gobierno comunista. Esta, más que otra razón, sería la causante del desenlace final de la República (2006: 25-29). De alguna manera, en el texto de Suárez Picallo, se responsabiliza al país natal de la situación mundial, insistiendo en la idea de que la República, de haberse mantenido como gobierno, habría posiblemente evitado el despliegue de un sistema tan poderoso como fue el nazifascista. Tal como se observa en "Carteles españoles", "Unidad republicano-socialista española" y "Efemérides españolas", la crítica a España se articula de acuerdo con una idealización de la República, en la que esta aparece definida por un gesto moral orientado a la lucha en pos de la libertad y la democracia. En relación con esto, Schwarzstein sostiene que los republicanos construían su identidad mediante, además del lazo con la tierra natal y el vínculo con la de acogida, el ennoblecimiento de la República como consecuencia inmediata de la idealización de la Guerra Civil y el bando izquierdista, configurándose así como defensores de una causa justa y generosa, de un ideal íntegro y honorable (2006: 64-67). Y a esto sigue la idealización de América como baluarte de la justicia al que hay imitar y apoyar para así reivindicar el sustento ideológico de un modelo que se opone a la barbarie fascista y respeta las intenciones de los pueblos independientes. Nuevamente surge la idea de una equivalencia simbólica entre la opresión de españoles y americanos, reforzada en la crónica "La República española y el día de las Américas", escrita en 1947, tres años después que la anterior: "Los españoles de América lucharon contra Fernando VII, desde 1810 a 1825. Los españoles de España lucharon, desde 1931 a 1939, contra los nietos de Fernando VII, que seguían manteniendo en España el régimen colonial que América había liquidado un siglo antes" (Suárez Picallo 2008: 274). Además de reiterar la noción de una colectividad enlazada por la resistencia a un enemigo común y opresor, Suárez Picallo apela a la historia para elevar la situación americana a una categoría ejemplar, pues, el que América haya podido alzarse en contra del imperio español para proclamar su independencia y mantenerla viene a significar, en el pensamiento del sujeto, que la zona republicana del país también puede hacerlo. $\mathrm{Y}$ en este sentido, se conforma la esperanza política de volver a instalar la República en la Península Ibérica, otorgándole fortaleza y continuidad a una base ideológica que encuentra la validez de sus instituciones en la experiencia americana, a pesar de haberse quedado con una connotación de fracaso en España tras el arribo del franquismo. Básicamente, el autor anuda al pueblo republicano y gallego, separado de la España imperialista y fascista, con el americano, en la búsqueda de una identidad que lo represente tanto a él, en cuanto sujeto desterrado, como también a todos aquellos que se posicionan en un lugar contrario al de las naciones europeas que quieren gobernar el mundo mediante el abuso y la destrucción. Y en este propósito radica la labor intelectual que ejerce largamente en los medios periodísticos sin abandonar jamás su articulación como exiliado gallego-republicano.

\section{A MODO DE CONCLUSIÓN}

Si bien las crónicas que conforman La feria del mundo. Crónicas desde Chile (1942-1956) abordan temáticas de variada índole, resulta factible establecer ciertas motivaciones recurrentes a lo largo de la obra. Una de ellas tiene que ver con el exilio republicano en América, especialmente el gallego, y la tensión latente entre el país de origen y el de acogida, pues, el sujeto se fragmenta entre ambos al no poder estar en el que considera suyo a causa de un régimen contrario a sus ideas, y tampoco completar la adaptación a tierra americana pese a verse representado en sus instituciones. De aquí derivan sus esfuerzos por identificar la opresión de América con la republicana frente a un enemigo común, perceptible en el 
pasado y en el presente, vinculado con el despotismo y el fascismo. La idea de Galicia, hermanada con la República, ocupa un lugar fundamental en el pensamiento del autor, ya que la concibe frustrada como nación libre e independiente tempranamente por los Reyes Católicos, quienes al igual que Franco, la despojan de su carácter plural, autónomo y democrático. Galicia, junto al resto de la zona republicana, funciona alegóricamente como la América lastimada por el poder español cimentado en la represión y el sometimiento forzado. No obstante, Suárez Picallo observa en Chile y en otros países del continente un ejemplo de liberación y mantenimiento de un sistema político asimilable al planteado por la
República, y ello le permite vislumbrar la posibilidad de recuperar la esperanza ideológica en España, acribillada por el franquismo y sus aliados europeos. Reiteradamente, en las crónicas aparece el interés por dar forma a una identidad colectiva, capaz de acoger a individuos de diferentes pueblos pero con el mismo ideal democrático en la resistencia a la tendencia fascista y totalitaria que se desarrolla en Europa en la primera mitad del siglo XX. Y es ese empeño el que convierte a Suárez Picallo en uno de los intelectuales gallegos en el exilio de mayor trascendencia en la tarea de repensar la nación y los caminos que dirijan a una democracia de orden mundial.

\section{REFERENCIAS BIBLIOGRÁFICAS}

Almonacid Zapata, Fabián (2004): "Españoles en Chile: Reacciones de la Colectividad frente a la República, Guerra Civil y Franquismo (1931-1940)", Revista Complutense de Historia de América, vol. 30, Madrid, pp. 149-185.

AlONSO GARCíA, Rosario (2006): "Política y propaganda del gobierno de la República española en el exilio, 1945-1962”, en Xosé Manoel Núñez Seixas y Pilar Cagiao Vila (eds.), O exilio galego de 1936: política, sociedade, itinerarios. Sada (A Coruña): Ediciós do Castro/Consello da Cultura Galega, pp. 79-101.

Alted VigiL, Alicia (2006): "En torno a la identidad del exiliado republicano de 1939 y sus culturas", en Xosé Manoel Núñez Seixas y Pilar Cagiao Vila (editores), O exilio galego de 1936: política, sociedade, itinerarios. Sada (A Coruña): Ediciós do Castro/Consello da Cultura Galega, pp. 69-77.

ANDERSON, Benedict (1993): Comunidades imaginadas. Reflexiones sobre el origen y la difusión del nacionalismo. México: Fondo de Cultura Económica.

Congreso da Emigración Galega (2006): 1856 - Banquete de Conxo - 1956: Primeiro Congreso da Emigración Galega: feito desde o 24 ao 31 de xulio de 1956 en Bos Aires (edición facsímile, $1^{\mathrm{a}}$ ed. 1956, Bos Aires). Santiago de Compostela: Consello da Cultura Galega, Arquivo da Emigración Galega.

Cornejo Serrano, Alex (2006): "El exilio galleguista de Ramón Suárez Picallo en Chile, 1940-1956", en Xosé Manoel Núñez Seixas y Pilar Cagiao Vila (eds.), O exilio galego de 1936: política, sociedade, itinerarios. Sada (A Coruña): Ediciós do Castro/Consello da Cultura Galega, pp. 375-394.

Boletín Oficial del Estado (2011): Resolución de 23 de noviembre de 2011, de la Dirección General de la Ciudadanía Española en el Exterior, por la que se establecen normas para la designación de miembros del Consejo General de la Ciudadanía Española en el Exterior. Boletin Oficial del Estado, 28/11/2011, no 286, p. 126.503.

Estrada Turra, Baldomero (2009): "República y Exilio Español en el fin del Mundo. Valparaíso, Chile”, Revista de Indias, vol. LXIX, no 245, pp. 95-122.

FERrer Mir, Jaime (1999): “Los españoles del Winnipeg”, en Winnipeg 60 años. Santiago de Chile: Centro Cultural de España.

(2004): Nómina actualizada de los españoles del Winnipeg. El barco de la Esperanza. 19392004. Santiago de Chile: Ediciones CAL SOGAS.

InSTITUTO GALEGO DE ESTADÍSTICA, http://www.ige.eu/web/index.jsp?idioma=gl. [visita 31/01/2012]

Norambuena, Carmen (1999): “El exilio español republicano. El aporte cultural”, en Winnipeg 60 años. Santiago de Chile: Centro Cultural de España.

Norambuena, Carmen y Garay, Cristián (2002): España 1939: Los Frutos de la Memoria. Disconformes y Exiliados Artistas e Intelectuales Españoles en Chile 1939-2000. Santiago: Instituto de Estudios Avanzados de la Universidad de Chile. 
NúÑEZ SeIXAS, Xosé Manoel (2006): "Itinerarios do desterró: sobre a especificidade do exilio galego de 1936”, en Xosé Manoel Núñez Seixas y Pilar Cagiao Vila (eds.), O exilio galego de 1936: política, sociedade, itinerarios. Sada (A Coruña): Ediciós do Castro/Consello da Cultura Galega, pp. 11-51.

Retamal, Julio, Celis Atria, Carlos y MuÑoz Correa, Juan Guillermo (1992): Familias Fundadoras de Chile. 1540-1600. Santiago de Chile: Zig-Zag.

SÁnChEZ EsPINOZA, María de las Nieves (1995): Chaguazoso. Una aldea gallega en Ultramar. Santiago de Chile: Ediciones Nueva Galicia (Red Internacional del Libro).

SCHWARZSTEIN, Dora (2006): "Migración, refugio y exilio: categorías, prácticas y representaciones", en Xosé Manoel Núñez Seixas y Pilar Cagiao Vila (eds.), O exilio galego de 1936: política, sociedade, itinerarios. Sada (A Coruña): Ediciós do Castro/Consello da Cultura Galega, pp. 53-67.

SuÁrez GonZÁLEZ, Andrés (2011): "La cultura gallega en Europa y su contexto en América Latina", en Osvaldo Carvajal Muñoz y Evelyn Soto Castillo (eds.),(Des)Encuentro Culturas Ibéricas: Haciendo hablar a la Diversidad (Actas del Primer encuentro de estudios ibéricos en Chile). Santiago de Chile: Universidad.

SuÁrez PicAllo, Ramón (2008): La Feria del Mundo. Crónicas desde Chile (1942-1956) [editores y compiladores: Edmundo Moure Rojas, Carmen Norambuena Carrasco; coordinadores de edición: Rosa Aneiros Díaz, Xosé López García]. Santiago de Compostela: Consello da Cultura Galega.

Thayer OJeDA, Luis (1989): Orígenes étnicos de Chile: Elementos étnicos, apellidos, familias. Santiago de Chile: Andrés Bello.

Villares, Ramón (1996): Historia da Emigración Galega a América. Santiago de Compostela: Xunta de Galicia. 